\title{
Síndrome de Burnout e sua relação com a ausência de qualidade de vida no trabalho do enfermeiro
}

Recebido em: 19/9/2011

Aceito em: 16/4/2012

\author{
Carolina Colleta Pereira Lopes ${ }^{1}$ \\ Taynah Piovesan Ribeiro' \\ Neudson Johnson Martinho
}

Trata-se de uma revisão integrativa, objetivando identificar no universo profissional dos enfermeiros a (in)existência de relação entre a síndrome de Burnout e a ausência de qualidade de vida no trabalho. O estudo ocorreu nos bancos de dados da Biblioteca Virtual de Saúde (Bireme), a partir de um quantitativo de 566 artigos com descritores relacionados à temática do estudo. Entretanto, destes, apenas 13 foram selecionados para compor a amostra deste estudo, por se enquadrarem nos critérios de inclusão estabelecidos. Faz-se necessário o desenvolvimento de mais pesquisas quanto a esse fenômeno investigado, tendo em vista a escassez de publicações identificadas. Concluímos, com esta pesquisa, que existe uma estreita relação entre síndrome de Burnout e a ausência de qualidade de vida no cotidiano profissional de enfermagem.

Descritores: Enfermagem, Esgotamento Profissional, Qualidade de Vida, Saúde do Trabalhador.

\section{Burnout syndrome and it's relation to the absence of quality of life of nurses at work}

This is integrative review aiming to identify in the universe of professional nurses the (in) existence of relationship between burnout syndrome and the lack of quality of work life. This study took place In the databases of the Healt Virtual Library (BIREME), where there was raised an amount of five hundred and sixty-six (566) articles with descriptors related to the topic. However, only thirteen (13) of these articles were selected to compose the sample of this study, because they fit the established inclusion criteria. It is necessary the development of more research on this subject, in view of the scarcity of publications identified. We conclude, from this research, that there is a close relation between burnout syndrome and lack of quality of life in daily nursing professional.

Descriptors: Nursing, Burnout, Quality of Life, Occupational Health.

\section{Sindrome de Burnout y su relación com la ausência de la calidad de vida de enfermeira em el trabajo}

Se trata de una revisión integradora, con el objetivo de identificar en el universo de profesionales de enfermería (en) la existencia de relaciones entre el síndrome de burnout y la falta de calidad de la vida laboral. El estudio se crió en las bases de datos de la Biblioteca Virtual de Salud (BIREME) desde una cantidad de quinientos sesenta y seis (566) artículos con los descriptores relacionados con el tema. Sin embargo, destos sólo trece 13) artículos fueron seleccionados para componer la muestra para este estudio por correspondieren a los criterios de inclusión establecidos. Es necesario desarrollar más investigación sobre este fenómeno investigado, en vista de la escasez de las publicaciones identificadas. Concluimos con esta investigación que existe una estrecha relación entre el síndrome de burnout y la falta de calidad de vida en la enfermería profesional diaria.

Descriptores: Enfermería, Agotamiento Profesiona, Calidad de Vida, Salud Ocupacional.

\section{INTRODUÇÃO}

$\mathrm{N}$ o desenvolvimento de seu trabalho, o enfermeiro vive situações de estresse no ambiente de trabalho, sendo alguns fatores contribuintes caracterizados por: número reduzido de profissionais de enfermagem em proporção à demanda, excesso em quantidade e variedade de atividades a serem conciliadas e executadas, dificuldade em delimitar os diferentes papéis entre as variadas classes da enfermagem, falta de reconhecimento do público assistido e dos próprios colegas, do grupo multidisciplinar e ainda da organização ${ }^{(1,2)}$.

Conceitua estresse como um esgotamento pessoal, sinônimo de cansaço, dificuldade, frustração, ansiedade, desamparo, desmotivação, conhecido por Burnout, fenômeno esse que interfere diretamente no indivíduo, mas não está necessariamente relacionado ao trabalho ${ }^{(2)}$.

Como se todos esses fatores não bastassem, há ainda que se mencionar o achatamento dos salários, que leva o profissional a mais de um vínculo empregatício, a uma carga horária mensal extremamente desgastante e os problemas "extras" do ambiente de trabalho, como, por exemplo, os de ordem familiar, que acarretam em acúmulo de desgaste emocional, ferindo, portanto, o conceito de qualidade de vida no trabalho ${ }^{(2)}$.

Burnout, no inglês, é definido como aquilo que deixou de funcionar por absoluta falta de energia. Aquele que chegou a seu limite, tendo prejudicado seu desempenho físico ou mental. É um processo que se inicia com excessivos e prolongados níveis de estresse (tensão) no trabalho ${ }^{(3)}$.

Entre as causas de Burnout nos enfermeiros, pode-se 
levantar o fato de que a maior parte do tempo de trabalho está relacionada ao contato direto com colegas de trabalho, pacientes e seus familiares. Há, geralmente, nessa relação interpessoal, o envolvimento nos sentimentos de tensão, ansiedade, medo e até mesmo de hostilidade encoberta ${ }^{(1)}$.

Nesta revisão, foi encontrado outro estudo, que corrobora o que está descrito pelos autores aqui citados, afirmando que a síndrome de Burnout atinge de forma geral os profissionais que lidam diretamente com pessoas. Isso abrange todo tipo de relações interpessoais, desde cliente/paciente, passando por colegas de trabalho, até chefes/supervisores ${ }^{(2)}$. O trabalhador perde o sentido de sua relação com o trabalho, as coisas já não têm mais importância e todo esforço parece ser inútil(4).

Existem três dimensões na síndrome que são independentes, mas podem ser associados: exaustão emocional: falta ou pouca energia para o trabalho, acompanhada de uma sensação de esgotamento emocional, que pode até ser somatizada; despersonalização: é o tratamento que se dá a pessoas e organizações como se fossem objetos, de forma desprovida de afetividade, sensibilidade, podendo até ser dissimulada. É natural, nessa dimensão, ansiedade, irritabilidade, falta de compromisso com resultados, alienação e conduta voltada para si, entre outras; falta de envolvimento no trabalho: há o sentimento de inadequação pessoal e profissional, em que o trabalhador se avalia de forma negativa, o que afeta a habilidade para realizar o trabalho $0^{(1,2,4)}$.

Com base na literatura e contextualizando-a à realidade vigente nos campos de práticas curriculares durante a graduação, observamos, tanto no nível hospitalar quanto na atenção básica que os enfermeiros demonstravam em suas falas em momentos informais, traços sugestivos de Burnout, sendo mais enfático na área hospitalar. Esse fenômeno observado nos despertou para o desenvolvimento deste estudo, o qual nos leva a questionar: não seria a ausência da qualidade de vida no trabalho um dos fatores associados à síndrome de Burnout ou fator desencadeador nos enfermeiros?

Nessa perspectiva, desenvolvemos esta pesquisa bibliográfica, objetivando identificar no universo profissional dos enfermeiros a (in)existência entre a síndrome de Burnout e a ausência de qualidade de vida no trabalho.

Consideramos relevante o estudo por tratar-se de uma temática vigente, a qual urge pela elaboração e implementação de estratégias que possam contribuir na melhoria da qualidade de vida no cotidiano profissional da enfermagem, tornando o cuidar/cuidado uma via de mão dupla na relação profissional $X$ cliente $\mathrm{X}$ organização de saúde.

\section{METODOLOGIA}

Este estudo faz parte da linha de pesquisa gestão de pessoas do Grupo de Pesquisa Educação e Formação em Saúde e Enfermagem - Gefor, da Faculdade de Enfermagem da Universidade Federal de Mato Grosso. Visa investigar uma temática relacionada ao estresse desencadeado nos enfermeiros em seu cotidiano profissional e a relação desse fenômeno com a qualidade de vida no trabalho através de uma revisão integrativa, tendo em vista que esse método de pesquisa permite a inclusão de estudos experimentais e não experimentais para uma compreensão completa do fenômeno analisado, combinando dados da literatura teórica e empírica, além de incorporar um vasto leque de propósitos: definição de conceitos, revisão de teorias e evidências e análise de problemas metodológicos de um tópico particular, vindo a atender os objetivos deste estudo. Para levantamento dos estudos sobre a temática investigada, foram estabelecidos como critérios de inclusão para análise que os artigos fossem escritos em língua portuguesa e publicados nos últimos cinco anos.

Os artigos foram pesquisados a partir de descritores cadastrados no Descritores em Ciências da Saúde (Decs), caracterizados por: esgotamento profissional, qualidade de vida, enfermagem e saúde do trabalhador. No processo de seleção dos descritores, foi encontrado o descritor em inglês Burnout, que, em português, equivale ao termo esgotamento profissional, o qual foi escolhido para ser utilizado neste estudo.

A seleção dos artigos para análise foi finalizada por um processo de filtragem, caracterizado pelo descarte dos artigos que se repetiam nas bases de dados, e, subsidiados nos critérios de inclusão, foram selecionados 13 artigos, apresentados no quadro de resultados, separados por banco de dados, periódico, idioma, ano de publicação, autor e título, organizados por congruência de resultados, os quais foram submetidos à análise analógica da relação entre Burnout e ausência de qualidade de vida no ambiente de trabalho do enfermeiro.

Esse levantamento de estudos ocorreu no período compreendido entre março e junho de 2010, na Biblioteca Virtual em Saúde - Bireme, onde estão indexadas as bases de dados: Banco de Dados de Enfermagem (BDENF) e Scientific Eletronic Library on-line-Brasil (Scielo), assim como no acervo literário da Biblioteca Central da Universidade Federal de Mato Grosso - UFMT.

\section{APRESENTAÇÃO DOS RESULTADOS}

No BDENF, foram encontrados 13 artigos com o descritor "Esgotamento Profissional", sendo utilizados dois para a análise, e, no Scielo, 15, sendo seis analisados.

Com o descritor "Qualidade de Vida e Enfermagem", foram encontrados no BDENF 130 artigos, dos quais dois foram selecionados para a análise; no Scielo, encontramos 27, porém nenhum contemplava os objetivos desta pesquisa.

Referente ao descritor "Saúde do Trabalhador", rastreamos 252 artigos no BDENF; no entanto, somente dois se enquadravam neste estudo para análise. Na base de dados Scielo, encontramos 129 estudos; desses, apenas três eram relevantes para esta pesquisa, totalizando 15 artigos levantados e selecionados para análise, porém apenas 13 foram utilizados, porque dois se repetiam em bases de dados diferentes e dois livros encontrados na biblioteca central da UFMT, por contemplarem o objeto deste estudo e contribuírem para compreensão dos fenômenos identificados.

$O$ trabalho é o meio que o homem dispõe para obter reconhecimento e prestígio social, além de atender a suas necessidades. Mas é também nesse universo que ele passa a maior parte de seu tempo, distanciando-se e/ou tendo de lidar com 


\begin{tabular}{|c|c|c|c|c|c|}
\hline FONTE/ORIGEM & PERIÓDICO/EDITORA & IDIOMA & ANO & AUTOR & TÍTULO \\
\hline \multirow[t]{2}{*}{ SCIELO } & Acta Paul. Enferm. & Português & 2008 & PASCHOALINI B, et al. & $\begin{array}{l}\text { Efeitos cognitivos e emocionais do estresse } \\
\text { ocupacional em profissinais de enfermagem }\end{array}$ \\
\hline & Rev. SBPH & Português & 2005 & ROSA C, CARLOTTO MS & $\begin{array}{l}\text { Síndrome de Burnout e satisfação no trabalho } \\
\text { em profissionais de uma instituição hospitalar }\end{array}$ \\
\hline \multirow[t]{5}{*}{ BDENF/SCIELO } & $\begin{array}{l}\text { Rev. Latino-Am. } \\
\text { Enfermagem }\end{array}$ & Português & 2005 & MUROFUSE NT, et al. & $\begin{array}{l}\text { Reflexões sobre estresse e Burnout e a relação } \\
\text { com a enfermagem }\end{array}$ \\
\hline & Rev. Psiquiatr. Clín. & Português & 2007 & TRIGO TR, et al. & $\begin{array}{l}\text { Síndrome de Burnout ou estafa profissional } \\
\text { e os transtornos psiquiátricos }\end{array}$ \\
\hline & Acta Paul. Enferm. & Português & 2009 & JODAS, DA, HADDAD, MCL & $\begin{array}{l}\text { Síndrome de Burnout em trabalhadores de enfermagem } \\
\text { de um pronto-socorro de hospital universitário }\end{array}$ \\
\hline & Ciênc. Saúde Coletiva & Português & 2005 & PIZZOLI, LML & $\begin{array}{l}\text { Qualidade de vida no trabalho: um estudo de } \\
\text { caso das enfermeiras do Hospital Heliópolis }\end{array}$ \\
\hline & $\begin{array}{l}\text { Rev. Ciênc. Méd. } \\
\text { Campinas }\end{array}$ & Português & 2006 & TALHAFERRO B, et al. & $\begin{array}{l}\text { Qualidade de vida da equipe de enfermagem da } \\
\text { central de materiais e esterilização }\end{array}$ \\
\hline \multirow[t]{2}{*}{ BDENF } & Rev. Enferm. Uerj & Português & 2009 & DALMOLIN, GL et al. & $\begin{array}{l}\text { O sofrimento moral dos profissionais de enfermagem } \\
\text { no exercício da profissão }\end{array}$ \\
\hline & Rev Gaúcha Enferm. & Português & 2008 & PANIZZON, C. et al. & $\begin{array}{l}\text { Estresse da equipe de enfermagem de } \\
\text { emergência clínica }\end{array}$ \\
\hline REVISTA EINSTEN & Einsten & Português & 2009 & SANTOS, F E. et al. & $\begin{array}{l}\text { Síndrome de Burnout em enfermeiros atuantes } \\
\text { em uma Unidade de Terapia Intensiva }\end{array}$ \\
\hline
\end{tabular}

Figura 1 - Apresentação de artigos e livros selecionados para compor amostra do estudo

dificuldades em suas relações pessoais e convívio social ${ }^{(5)}$. Como consequência da velocidade das mudanças no mundo do trabalho e da dificuldade de adaptação do ser humano a essas mudanças, surgem reações como insatisfação generalizada com o modo de vida, tédio, angústia, ambiguidades, ansiedade, despersonalização, frustração e alienação no trabalho, entre outros.

Buscando construir um diálogo entre os autores dos artigos selecionados para análise, dispomos os mesmos relacionando qualidade de vida e Burnout para visualizar possibilidade de influência entre os fenômenos.

Segundo Paschoalini ${ }^{(6)}$, os fatores desencadeantes de estresse no trabalho são condições e organização de trabalho, sobrecarga laboral por dupla jornada e realização de funções cujas demandas são inferiores às qualificações do profissional, desencadeando nos enfermeiros sentimento de frustração profissional.

Rosa e Carlotto ${ }^{(1)}$ discordam em parte, trazendo as relações interpessoais negativas, além de fatores organizacionais, como falta de inovação e estímulo, pouca autonomia, dificuldade de relação com superiores e clientes, pressões burocráticas e falta de feedback da organização como as causas mais vigentes de frustração e Burnout.
Pizzoli complementa citando ausência de reconhecimento pelo trabalho, falta de plano de carreira, deficiência de comunicação e incompatibilidade do salário com a função (valor abaixo do mercado) como uma associação que coloca em risco a motivação e a satisfação do enfermeiro, podendo interferir na produtividade e na qualidade do serviço(7).

Nessa perspectiva, ressalta-se que os agentes estressores ocupacionais podem produzir ainda efeitos cognitivos e emocionais associados. O desenvolvimento de disforia, isto é, o comprometimento leve do humor produz prejuízo na orientação temporal, evocação tardia e diminuição das funções cognitivas. Existe uma relação entre a diminuição da orientação temporal e da memória, em associação com indicadores de depressão(6).

Detectamos que existem fatores internos e externos ao trabalho desencadeantes da depressão. Os fatores internos, inerentes ao processo de trabalho, são: os diferentes setores de atuação profissional, com suas peculiaridades, turno de trabalho, tipo de serviço, problemas na escala, a assistência em si a clientes, insegurança, conflito de interesses e as estratégias de enfrentamento desenvolvidas. São considerados externos ao trabalho fatores relacionados direta ou indiretamente ao 
indivíduo, como: sexo, idade, carga de trabalho doméstico, suporte e renda familiar, estado de saúde geral do trabalhador e características pessoais, como possíveis desequilíbrios químicos cerebrais, personalidade, vulnerabilidade genética e reação a eventos situacionais ${ }^{(8)}$.

Os resultados de um estudo apontam o enfermeiro como o profissional que vivencia com maior intensidade o estresse, sendo que os sinais no princípio da vida profissional sugerem prejuízos leves e alertam para o agravamento com a cronicidade ${ }^{(6)}$.

Existem estudos que enfatizam que, no princípio da carreira, os profissionais jovens, por não ter contato suficiente com as atividades da profissão, passam por muitas frustrações até aprenderem a lidar com as demandas do trabalho e com os sentimentos em relação ao mesmo ${ }^{(1,6)}$.

Nesse sentido, a síndrome de Burnout surge entre esse público primeiramente devido aos sentimentos com relação a mudanças sociais, às poucas habilidades e recursos, treinamento inadequado e falta de consciência quanto aos riscos para o desenvolvimento dessa síndrome. Esses motivos são considerados agravantes típicos do profissional jovem, que, em busca de melhores condições profissionais e financeiras, se expõe a jornadas de trabalho mais extensas, à adaptação a ambientes de trabalho diferentes, agregados à inexperiência e ao distanciamento da clientela pelo aumento da demanda.

De outro modo, no ambiente hospitalar há a tendência de investimento em estrutura física e na estética das instalações com fins de mercado, em detrimento do investimento em qualidade para a realização do trabalho dos profissionais( ${ }^{(1)}$. $O$ trabalhador insatisfeito com suas atribuições, somadas à forma de supervisão a qual estão sujeitos, ao ambiente físico de trabalho, aos benefícios e políticas hospitalares voltadas para os clientes e não para o trabalhador, pode vir a afastar-se dos usuários, perdendo o sentimento de envolvimento profissional, como forma de reação e enfrentamento à condição estressante.

Elias e Navarro ${ }^{(9)}$ defendem que os profissionais expostos a condições de trabalho precárias aliadas a suas condições de vida têm aumentadas as chances de chegar à síndrome de Burnout. Daí percebe-se a relação entre Burnout e ausência de qualidade de vida no trabalho.

Fernandes(10:13) complementa Elias e Navarro(9), enfatizando que não se pode falar em qualidade de produtos e serviços se aqueles que vão produzi-los não têm qualidade de vida no trabalho.

Portanto, prestar cuidados em condições adversas de trabalho, em consequência da obrigação de trabalhar por necessidade de sobrevivência, forma o cotidiano profissional de muitos profissionais enfermeiros, levando-os a desenvolver Burnout, o qual está relacionado à inexistência de qualidade de vida no trabalho (QVT), de modo específico no ambiente hospitalar ${ }^{(11)}$.

Em busca de qualidade de vida, que na sociedade atual vem associada principalmente ao poder de consumo, o profissional enfermeiro com vistas à produção supervalorizada pelo sistema capitalista se submete à falta de qualidade de vida no trabalho, para ter garantido seu sustento e a manutenção do padrão de vida adotado(2,11,12).
A Organização Mundial da Saúde - OMS reforça essa ideia: "Qualidade de vida é a percepção do indivíduo de sua posição na vida, no contexto, na cultura e sistema de valores nos quais ele vive e em relação a seus objetivos, expectativas, padrões e preocupações"(12:199).

O processo de globalização e implementação de políticas de recorte neoliberal tem impactado no trabalho de enfermagem, no sentido de provocar a crescente terceirização e exclusão dos trabalhadores do trabalho formal, comprometendo a garantia dos direitos sociais, elevando a rotatividade da força de trabalho, aumento do desemprego, contribuindo para o surgimento de processos destrutivos no mundo do trabalho da enfermagem, causando descontentamento e desencanto nos trabalhadores e, sobremaneira, comprometendo a qualidade de vida no trabalho (QVT) ${ }^{(13)}$.

Nessa perspectiva, os trabalhadores da enfermagem convivem com a dialética das contradições existentes entre os processos potencializadores (protetores, geradores de satisfação, saúde) e os destrutivos presentes nas diversas ambiências de trabalho e na vida social. Esse processo dialético ocorre simultaneamente, sendo que a QVT é gerada quando se trabalha os aspectos potencializadores (protetores, geradores de vida) em oposição aos destrutivos presentes e mais facilmente percebidos no mundo do trabalho ${ }^{(13)}$

Não podemos falar de QVT sem compreendê-la como sinônimo de saúde do trabalhador, partindo do conceito ampliado de saúde, o qual transcende a simples ausência de doenças. Nesse sentido, a QVT dos trabalhadores de enfermagem pressupõe apreender a saúde desses sujeitos no contexto histórico e social, pois essa é uma construção social.

O processo saúde-doença no trabalho é concebido como nexo biopsíquico, o qual se manifesta a partir das atividades no trabalho. É expresso pelo desgaste sofrido pelos trabalhadores de enfermagem, provocado pela exposição às cargas de trabalho, processos de valorização e valoração aos quais os mesmos são submetidos. É perceptível como a relação com o trabalho como categoria social se expressa no corpo do trabalhador, fruto de sua saúde mental, consequência de fatores negativos desencadeados sobre sua autoestima e autoimagem ${ }^{(1)}$.

\section{CONSIDERAÇÕES FINAIS}

Identificamos que síndrome de Burnout em muitos casos se relaciona com a qualidade de vida no trabalho, a qual tem sido um desafio constante para muitos enfermeiros que assumem cargos de gerência nos serviços de enfermagem, assim como para os próprios enfermeiros assistenciais e a equipe em geral.

Esse fenômeno (Burnout), hoje objeto de estudos em todo o mundo, tem elevada importância por não ser apenas um problema de cunho psicológico, mas também por envolver aspectos sociais, gerenciais, econômicos e culturais. Preconizado internacionalmente como objeto de intervenções no processo de Gestão de Pessoas nas diversas organizações de trabalho, porém, verifica-se implicitamente nos artigos investigados a existência de nós críticos que se caracterizam pela falta de habilidade e disposição dos gestores quanto à 
percepção e intervenção sobre o mesmo.

Nesse sentido, é necessário que as instituições disponham de uma infraestrutura adequada de trabalho com ambiente agradável e humanizado, dimensionamento adequado de profissionais, acesso e comunicação clara com supervisores enfim, políticas organizacionais que contemplem a qualidade de vida no trabalho.

Este estudo nos possibilitou identificar que o relacionamento interpessoal negativo na equipe de enfermagem e dessa com a equipe de saúde é um dos fatores contribuidores para o surgimento da síndrome de Burnout, relações essas que perpassam por relações de poder e desrespeito às competências dos enfermeiros em relação aos demais membros da equipe, levando-nos a inferir que as atividades que envolvam o processo de comunicação e percepção da interdisciplinaridade na equipe devem ser objetos de avaliações e implementações de estratégias que deem sentido à importância do trabalho em equipe a partir da uma comunicação ativa nas relações interpessoais no ambiente de trabalho.

Quanto ao enfrentamento dos agentes estressores que levam à síndrome de Burnout profissionais já diagnosticados, as organizações podem promover ações educativas sobre as possíveis formas de enfrentamento e viáveis mudanças nas condições do trabalho, lotação de funcionários conforme aptidão e afinidade com as diversas áreas do cuidar, e estimular em parceria com instituições de ensino superior o desenvolvimento de ações em educação permanente e continuada, além de pesquisas que permeiem os processos de gerir pessoas e talentos, elucidando possibilidades para redução desse fenômeno, considerado hoje de cunho socioorganizacional.

Nessa perspectiva, entendemos ser imprescindível o diálogo entre o setor de gestão de pessoas das organizações de saúde com enfermeiros envolvidos na administração dos serviços de enfermagem e com os demais colaboradores da equipe de saúde como algo que deve ser priorizado e utilizado como ferramenta fundamental para diagnóstico, avaliação e melhoria das condições de trabalho, de ajuda ao enfrentamento das situações desencadeadoras de Burnout, contribuindo para a prevenção de agravos psicológicos entre trabalhadores da equipe de enfermagem e, consequentemente, redução de prejuízos organizacionais.

Inferimos, após estas leituras crítico-analíticas, que existem fragilidades no processo de gerir pessoas, levando em consideração que a síndrome de Burnout pode apresentar menor incidência quando o setor de gestão de pessoas atuar de forma efetiva e coparticipativa nas organizações de saúde, merecendo atenção peculiar o serviço de enfermagem, por esse demandar maiores situações de estresse, por interagir por mais tempo e mais diretamente com o paciente, família e equipe multidisciplinar de saúde.

É importante ressaltar a necessidade do desenvolvimento de pesquisas quanto a essa síndrome, pois é factual a escassez de artigos científicos sobre a mesma, de modo peculiar em língua portuguesa. Nesse sentido, se faz necessário que outros estudos sejam elaborados para que esse fenômeno seja mais bem conhecido e identificado precocemente nas instituições de saúde e sofra intervenções imediatas, possibilitando que o trabalho de enfermagem seja percebido como algo prazeroso e (re)encantando a cada dia.

Enfim, a categoria profissional tem de perceber e compreender o espaço de trabalho da enfermagem como micropolítico, sobre o qual são reproduzidas as políticas sociais e de saúde. Espaço esse possível de transformações, desde que os enfermeiros (re)pensem e lutem por seu poder e atitude política, e em uma luta coletiva busquem mostrar e viabilizar na sociedade mudanças concretas nos cenários laborais da enfermagem no que tange a QVT e saúde do trabalhador ${ }^{(13)}$.

\section{Referências}

1. Rosa C, Carlotto MS. Síndrome de Burnout e satisfação no trabalho em

profissionais de uma instituição hospitalar. Rev SBPH. 2005;8(2):1-15.

2. Murofuse NT, Abranches SS, Napoleão AA. Reflexões sobre estresse e

burnout e a relação com a enfermagem. Rev Latinoam Enferm. 2005;13(2):255-

61.

3. Trigo TR, Teng CT, Hallak, JEC. Sindrome de Burnout ou estafa profissional e

os transtornos psiquiátricos. Rev Psiquiatr Clín (São Paulo). 2007;34(5):223-33.

4. Santos FE, Alves JÁ, Rodrigues AB. Síndrome de Burnout em enfermeiros

atuantes em uma unidade de terapia intensiva. Einstein. 2009;7(1):58-63.

5. Talhaferro B, Barbosa DB, Domingos NAM. Qualidade de vida da equipe

de enfermagem da central de materiais e esterilização. Rev Ciênc Méd.

2006;15(6):495-506.

6. Pachoalini B, Oliveira MM, Frigério MC, Dias ALRP, Santos FH. Efeitos

cognitivos e emocionais do estresse ocupacional em profissionais de

enfermagem. Acta Paul Enferm. 2008;21(3):487-92.

7. Pizzoli LML. Qualidade de vida no trabalho: um estudo de caso das

enfermeiras do hospital Heliópolis. Ciênc Saúde Coletiva. 2005;10(4):1055-62.
8. Manetti ML, Marziale MHP. Fatores associados à depressão relacionada ao

trabalho de enfermagem. Estud Psicol. 2007;12(1):79-85.

9. Fernandes E. Qualidade de vida no trabalho: como medir para melhorar. 3a

ed. Salvador: Casa da Qualidade; 1996.

10. Elias MA, Navarro VL. A relação entre o trabalho, a saúde e as condições de vida: negatividade e positividade no trabalho das profissionais de enfermagem de um hospital escola. Rev Latinoam Enferm. 2006;14(4):517-25.

11. Jodas $D A$, Haddad MCL. Síndrome de Burnout em trabalhadores de

enfermagem de um pronto socorro de hospital universitário. Acta Paul Enferm. 2009;22(2):192-7.

12. Fleck MPA, Lousada S, Xavier M, Chamovich E, Vieira G, Santos L, et al. Aplicação da versão em português do instrumento de avaliação de qualidade de vida da Organização Mundial da Saúde (WHOQOL-100). Rev Saúde Pública. 1999;33(2):198-205.

13. Felli VEA, Tronchin MR. A qualidade de vida no trabalho e a saúde do trabalhador de enfermagem. In: Kurgant P, coordenador. Gerenciamento em enfermagem. Rio de Janeiro: Guanabara Koogan; 2005. p. 89-107. 